\title{
USO DE ENXERTOS RENAIS DE DOADORES FALECIDOS COM LESÃO AGUDA POR RABDOMIÓLISE É POSSÍVEL?
}

\author{
Using kidney grafts from deceased donors with acute kidney injury due to \\ rhabdomyolysis. Is it possible? \\ Fernanda Folla Pompeu Marques', Emanuela Yumi Fugisawa de Mello1, Tiago Genzini de Miranda1, \\ Natallia Meira Gonsalez², Isabela Pereira Blanco ${ }^{2}$, Marcio Paredes ${ }^{3}$, Leon Alvim³, \\ Celia Watanabe ${ }^{3}$, Francisco Sergi ${ }^{3}$, Juan Branez ${ }^{3}$, Marcelo Perosa ${ }^{3}$
}

\section{RESUMO}

Introdução: Transplante de rim (TR) é a terapêutica de escolha para doença renal terminal. A universal desproporção entre demanda para TR e doadores disponíveis exige o desenvolvimento de novas estratégias para aumento da oferta de enxertos renais. Entre estas, citam-se o aumento de TR intervivos e o uso de doadores falecidos com critérios expandidos (DCE). Enxertos com lesão renal aguda (LRA) representam uma das situações de DCE, e sua utilização persiste sendo tema controverso diante da incerteza da reversão da lesão renal pós-TR. Rabdomiólise (RB) é a causa de 7 a 10\% dos enxertos com LRA e decorre da liberação de mioglobina na circulação e consequente lesão tubular por mecanismos diversos. Objetivos: Motivados por oferta recente de enxerto renal com RB e posterior não autorização de uso pela Organização de Procura de Órgâos, os autores objetivaram revisar a literatura sobre a viabilidade de TR de doador falecido com RB. Métodos: Revisão da literatura do banco de dados Medline usando descritores específicos, no período de 2000 a 2018. Resultados: Sete artigos demonstraram experiências com total de 51 TR de enxertos com RB. Dos 29 doadores com RB, 20,7\% encontravam-se em hemodiálise no momento da doação e a mediana da creatinina era de $3,9 \mathrm{mg} / \mathrm{dl}$. TR com doadores em RB apresentaram função retardada do enxerto em $35,3 \%$ dos casos, mas um mês após a cirurgia, os rins mostraram recuperação total e função normal, confirmadas por biópsia. Conclusão: Não há critérios brasileiros bem estabelecidos para uso de enxertos renais com RB, mas estudos atuais demonstram bons resultados e recuperação da função renal na grande maioria desses casos. Por enquanto, o aproveitamento de enxertos renais com RB deveria ficar a critério e julgamento de cada equipe.

Descritores: Transplante Renal, Rabdomiólise, Doação de Órgãos, Lesão Renal Aguda.

Instituições:

1 Faculdade de Ciências Médicas e da Saúde da Pontifícia Universidade Católica de São Paulo - São Paulo/SP

2 Faculdade de Medicina do ABC - Santo André/SP

3 Grupo Hepato - Departamento de Transplantes de Órgãos Abdominais do Hospital Leforte - São Paulo/SP

\section{Correspondência:}

Fernanda Folla Pompeu Marques.

Rua Francisco Pessoa, 690, casa 30, CEP $05727-230$ - São Paulo/SP Tel: (11) 99557-5586

E-mail: fernandamarques23@hotmail.com

\section{INTRODUÇÃO}

Transplante de rim (TR) é a terapêutica de escolha para doença renal terminal. A universal desproporção entre a necessidade de transplantes e número de doadores disponíveis faz com que milhares de pacientes acumulem-se nas filas de espera para TR, aumentando anualmente a mortalidade em lista para transplante (TX). Nos Estados Unidos, por exemplo, cerca de 90 mil pacientes estão na lista para TR, com tempo médio de espera de vários anos e mortalidade anual de 4500 pacientes enquanto aguardam o transplante. ${ }^{1}$ No Brasil, até dezembro de 2016, registravam-se 21264 pacientes na lista nacional de TR, sendo que 5492 procedimentos 
foram realizados nesse mesmo ano, capazes, portanto, de retirar apenas um quarto dos pacientes da lista de transplantes. ${ }^{2}$ Essa realidade exige estratégias para aumentar o número e aproveitamento de doadores renais vivos ou falecidos. Entre elas, inclui-se o uso progressivo de doadores falecidos com critérios expandidos. ${ }^{3}$

Doadores falecidos com lesão renal aguda são cada vez mais aproveitados e utilizados para TR. Entre as causas de LRA em doadores falecidos inclui-se a rabdomiólise, evento que ocorre em pacientes com grandes lesões musculares e teciduais devido a trauma. Apesar da maioria dos pacientes vivos recuperarem função renal após evento de RB, os doadores renais com esse quadro têm sido habitualmente descartados ou recusados pelas equipes diante da incerteza de reversão da lesão renal após transplante. ${ }^{4}$

Motivados pela oferta de enxerto renal de doador falecido com RB pela Central de Transplantes à nossa equipe, e, posteriormente, cancelamento da captação desses rins pela Organização de Procura de Órgãos por julgarem-nos impróprios para transplantes, e em vista de não haver critério bem definido para oferta e uso de doadores renais nessas condições em nosso país, decidiu-se revisar esse importante tema na literatura.

\section{MÉTODO}

Realizou-se extensa revisão de literatura do banco de dados Medline, com uso dos descritores: "kidney transplantation", "rhabdomyolysis", "tissue donor" e "acute kidney injury", no período de 2000 a 2018. Aceitaram-se todos os tipos de artigos em inglês e português.

\section{RESULTADOS}

Sete artigos foram encontrados e os principais dados estão ilustrados na Tabela 1. Esses estudos acumularam experiência com total de 51 TR de enxertos com RB. Dos 29 doadores com RB, 20,7\% encontravam-se em hemodiálise no momento da doação e a mediana da creatinina era de $3,9 \mathrm{mg} / \mathrm{dL}$. TR com enxertos em RB apresentaram função retardada do enxerto em $35,3 \%$ dos casos, mas um mês após a cirurgia, os rins mostraram recuperação total e função normal, confirmadas por biópsia.

Três artigos destacaram-se pelo maior número de receptores e tempo maior de seguimento. Mekeel et al, em 2009, relataram TR em dez pacientes com enxertos de doadores em RB; com média de creatinina dos doadores no último exame pré-TX de $4 \mathrm{mg} / \mathrm{dL}$ e, após quatro meses do transplante, os receptores atingiam média de $1,05 \mathrm{mg} / \mathrm{dL}$. Joshi et al descreveram quatro TR de doadores com RB com níveis de creatinina pós TX de $1,1 \mathrm{md} / \mathrm{dL}$. Chen et al, em 2017, publicaram estudo com maior número de pacientes, incluindo 15 doadores com média de creatinina sérica de $3,6 \mathrm{mg} / \mathrm{dL}$. Dos 30 receptores, seis necessitaram de diálise pós TX.

Tabela 1 - Estudos da Literatura com uso de Enxertos Renais em Rabdomiolise

\begin{tabular}{|c|c|c|c|c|c|c|c|c|}
\hline Autor & Ano & $\begin{array}{c}\text { Doadores } \\
\text { Utilizados } \\
\mathbf{N}\end{array}$ & $\begin{array}{c}\text { Doadores } \\
\text { em diálise } \\
\mathbf{N}\end{array}$ & $\begin{array}{l}\text { Creatinina } \\
\text { média dos } \\
\text { doadores } \\
(\mathrm{mg} / \mathrm{dL})\end{array}$ & $\begin{array}{c}\text { Receptores } \\
\text { transplantados } \\
\mathbf{N}\end{array}$ & $\begin{array}{c}\text { Receptores } \\
\text { com Diálise Pós- } \\
\text { Transplante }\end{array}$ & $\begin{array}{l}\text { Tempo médio } \\
\text { de diálise Pós- } \\
\text { transplante }\end{array}$ & $\begin{array}{l}\text { Creatinina média } \\
\text { final nos receptores } \\
\text { (mg/dL) }\end{array}$ \\
\hline Thomusch et al & 2001 & 1 & 0 & 4,7 & 2 & 2 & 17 dias & Após 3 meses: 0,9 \\
\hline Mekeel et al & 2009 & 7 & 1 & 4 & 10 & 3 & 10 dias & Após 12 meses: 1,3 \\
\hline Klein et al & 2019 & 1 & 0 & 13,1 & 2 & 1 & 11 dias & Após 4 meses: 1,05 \\
\hline Joshl et al & 2012 & 3 & 2 & 3,9 & 4 & 3 & 9 dias & $\begin{array}{l}\text { Após } 3 \text { meses: } 2 \\
\text { Após } 1 \text { mês: 1,1 }\end{array}$ \\
\hline Phair et al & 2013 & 1 & 0 & 1,4 & 2 & 2 & Não relatado & Após 32 meses: 1.1 \\
\hline Leyking et al & 2014 & 1 & 1 & 2,16 & 1 & 1 & 2 dias & Após 7 meses: 1,5 \\
\hline Chen et $\mathrm{Al}$ & 2017 & 15 & 2 & 3,6 & 30 & 6 & 7 dias & Após 1 mês: 1,02 \\
\hline Total & & 29 & 6 & $\begin{array}{c}3,9 \\
\text { (mediana) }\end{array}$ & 51 & 18 & $\begin{array}{l}10 \text { dias } \\
\text { (média) }\end{array}$ & Não relatado \\
\hline
\end{tabular}


Fernanda Folla Pompeu Marques, Tiago Genzini de Miranda, Emanuela Yumi Fugisawa de Mello, Natallia Meira Gonsalez, Isabela Pereira Blanco, Marcio Paredes, Leon Alvim, David Machado, Celia Watanabe, Francisco Sergi, Juan Branez, Marcelo Perosa

Os estudos variaram quanto ao tempo de seguimento pós-transplante, de três a 32 meses, todos alcançando níveis normais de creatinina sérica.

\section{DISCUSSÃO}

Existe grande desproporção entre o número de pessoas na fila para transplantes e doadores renais disponíveis. TR é o tratamento de escolha para pacientes em estágio final de doença renal, capaz de melhorar a qualidade de vida e reduzir os índices de mortalidade comparados aos pacientes mantidos em terapia renal substitutiva. ${ }^{5,6} \mathrm{Em}$ 2015 no Reino Unido, havia mais de cinco mil pacientes na lista de espera para TR, com média de menos de três mil transplantes realizados anualmente. ${ }^{5}$ No Brasil, o cenário em 2017 confirma tal desproporção. Em setembro de 2017, havia 20.595 pessoas ativas na lista para TR, e durante todo o ano foram realizados 4429 procedimentos. Nesse mesmo ano, 3559 pacientes faleceram na fila. ${ }^{2}$

Por essa razão, há necessidade de ampliarem e flexibilizarem os critérios de aceitação de enxertos renais através de DCE, que inclui doadores idosos (acima de 60 anos), com morte por parada circulatória e doadores com LRA. O uso de DCE foi proposto nos anos 2000, incluindo-se também doadores com comorbidades e aqueles com LRA, já que esse evento apresenta grande potencial de recuperação de função renal pós-transplante. ${ }^{7}$

A LRA é uma síndrome caracterizada por perda aguda da função renal e possui diferentes etiologias, sendo sua causa multifatorial. Afeta a maioria dos pacientes hospitalizados e as causas mais comuns são sepsis e hipovolemia. $^{8}$

Uma das razões para não aproveitamento de enxertos renais, especialmente de doadores jovens, é a LRA, pelo receio de não funcionamento ou baixa sobrevida do enxerto. Contrário a isso, Kumar et. al. demonstraram que sob critérios rigorosos, como doador abaixo de 50 anos, sem história pregressa de doença renal, hipertensão ou diabetes e biópsia pré-transplante normal do enxerto renal, a sobrevida dos enxertos foi comparável ao grupo de doadores ideais.

Após o período de diálise pós-transplante, a função renal de todos os enxertos utilizados com LRA foi semelhante a enxertos de doadores ideais dos seis meses aos três anos pós-operatórios, indicando que esses órgãos alcançam boa função no período pós-transplante. O estudo demonstra que a necessidade de diálise pós- transplante é mais elevada com o uso de enxertos renais de doadores com LRA, fato que não afeta os índices de rejeição ou função do enxerto em longo prazo. ${ }^{9,10}$

Uma das causas de LRA é RB, síndrome caracterizada por disfunção muscular com ruptura do sarcolema e consequente estravazamento de creatina quinase (CK), creatinina e, principalmente, mioglobina para a corrente sanguínea. ${ }^{11} \mathrm{~A} R \mathrm{R}$ afeta quase $10 \%$ de todos os pacientes com LRA nos Estados Unidos, e pode ser causada por traumas, exercícios exacerbados, imobilização prolongada, uso de cocaína, choque elétrico e raramente por uso de estatina. ${ }^{4,6}$ Descrevem-se também outras causas como afogamento, desidratação e sepse. ${ }^{12}$

A fisiopatologia da RB envolve ruptura de células musculares e liberação de mioglobina, que pode causar danos aos rins por diversos mecanismos. O aumento dos níveis de mioglobina e sua precipitação nos túbulos causa obstrução do lúmen e reduz a filtração glomerular. ${ }^{13}$ Esse processo ganha maiores dimensões pela desidratação e vasoconstrição renal. O estado de desidratação é comum e geralmente acompanha o quadro, pois o dano muscular grave determina sequestro de água para o espaço extracelular, causando importante depleção do volume intravascular. ${ }^{6} A$ vasoconstrição renal é consequência da ativação da cascata de citocinas via grupo heme da mioglobina. Além disso, a degradação intratubular da mioglobina causa liberação de espécies reativas de oxigênio que causam dano tissular oxidativo. ${ }^{6}$

O diagnóstico de RB pode ser feito pelos níveis de CK. Existem dois tipos de frações dessa enzima: MM e $\mathrm{MB}$, de origem muscular e cardíaca, respectivamente. Assume-se que níveis cinco vezes acima do normal ou maiores que $1.000 \mathrm{U} / \mathrm{L}$, sejam compatíveis com RB. ${ }^{6,14} \mathrm{~A}$ apresentação mais comum se dá por mialgia, fraqueza e mioglobinúria, embora sejam sintomas inespecíficos presentes em apenas metade dos casos. ${ }^{11}$ A terapia da RB inclui tratamento da causa de base, expansão volêmica, manitol intravenoso e alcalinização da urina, sendo necessária diálise, dependendo de cada caso. ${ }^{6,14,15}$

Muitos estudos concordam e demonstram que LRA e RB não deveriam ser contraindicação para doação renal. ${ }^{6,12,14}$ Mesmo que os pacientes possam apresentar função tardia do enxerto, após um mês de TR os enxertos geralmente demonstram total recuperação e

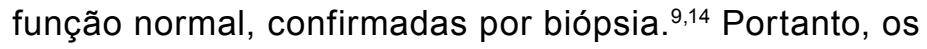
resultados mostram-se promissores e representam boa opção para ampliar o pool de doadores. ${ }^{6}$ 
A maioria da literatura baseia-se em relatos de caso ou de pequenas séries com uso de rins de doadores falecidos com RB. Um deles descreveu doador de 21 anos com dano cerebral por overdose medicamentosa e intoxicação alcoólica. Tal relato demonstrou doador anúrico e com altas taxas de CK (80 mil U/L), com evolução satisfatória e com $0,72 \mathrm{mg} / \mathrm{dL}$ de creatinina sérica, um ano e cinco meses pós-TR. ${ }^{7}$ Em 2013, outro relato demonstrou uso de enxerto renal de doador falecido de dois anos de idade, com morte causada por afogamento de 45 minutos. O doador apresentava creatinina de $1,4 \mathrm{mg} / \mathrm{dL}$, CPK de $76.826 \mathrm{U} / \mathrm{L}$ e o tempo de isquemia fria foi de 40 horas. Os rins foram mantidos em perfusão com máquina pulsátil (MP) e ambos foram transplantados em receptores idosos com índice de massa corpórea normal. Houve função tardia do enxerto e necessidade de diálise até os dias 14 e 29; porém, sete meses após o TR, os níveis de creatinina estabilizaram em $1,5 \mathrm{mg} / \mathrm{dL}$. Assim, os autores deste relato, como os demais, encorajam a utilização de rins provenientes de doadores falecidos com RB. ${ }^{12}$

O seguimento mais longo documentado foi publicado em 2012 em estudo retrospectivo de sete anos, que envolveu quatro TR com doadores em RB. Todos os rins foram submetidos a isquemia fria e MP por 33 e 28 horas em média, respectivamente.

Em um dos receptores, descreve-se diminuição dos níveis de mioglobina de $157.000 \mu \mathrm{g} / \mathrm{L}$ para $3.210 \mu \mathrm{g} / \mathrm{L}$ em 18 horas. Este artigo demonstrou que os níveis de creatinina dos receptores transplantados ficaram abaixo de $1,5 \mathrm{mg} / \mathrm{dl}$ após um mês em todos os casos, e após 72 meses de seguimento, foi demonstrada creatinina de $1,2 \mathrm{mg} / \mathrm{dL}$ nos pacientes. ${ }^{6}$ Sendo assim, concluíram que potenciais doadores com LRA secundária a RB não deveriam ser descartados. A biópsia renal é necessária para excluir outras causas da perda da função renal. Tais rins costumam ter maior ocorrência de função tardia do enxerto, mas após algumas semanas mostram normalização da função renal. ${ }^{14}$
O uso da MP ocorreu em três dos sete artigos revisados. ${ }^{6,12,15}$ Estabeleceu-se que ela confere melhor e mais rápida função do enxerto quando comparada à preservação estática, devido, provavelmente, ao fato de remover os metabólitos tóxicos e eliminar cristais de mioglobina nos rins a serem transplantados. ${ }^{6}$

O estudo mais atual e com maior número de casos provém de um centro chinês que incluiu série clínica entre 2012 a 2016. Um total de 15 doadores com LRA por RB foi analisado, com pico de CK de $15.569 \mathrm{U} / \mathrm{L}$ e creatinina média de $3,6 \mathrm{mg} / \mathrm{dL}$. Esses 15 doadores foram aproveitados para TR em 30 pacientes e comparados a grupo controle de TR com doadores ideais, não havendo diferença significativa Estudos da Literatura com uso de Enxertos Renais em Rabdomiolise nos resultados após dois anos. Os rins com rabdomiólise tinham aparência descorada e escura macroscopicamente e o exame patológico confirmou que o acúmulo de mioglobina nos túbulos era a causa do escurecimento. O critério de biópsia para aceitar tais órgãos foi que o comprometimento glomerular deveria ser menor que $50 \% .^{15}$

\section{CONCLUSÃO}

No Brasil, não há critérios definidos para alocação de doadores falecidos com RB, ficando o uso de enxertos renais nesses casos a critério de cada equipe. Apesar de ainda acumular poucos casos, a literatura mostra bons resultados iniciais e suporta o uso de tais enxertos, desde que a biópsia renal pré-transplante seja favorável. Como a prevalência de RB é elevada entre doadores falecidos com LRA de cerca de 10\%, geralmente acometendo doadores jovens de boa qualidade e vítimas de acidentes ou traumas, o aproveitamento desses enxertos, principalmente se preservados em MP, pode ser outra estratégia de expansão do pool de doadores e redução da fila de espera para TR. ${ }^{5}$ 
Fernanda Folla Pompeu Marques, Tiago Genzini de Miranda, Emanuela Yumi Fugisawa de Mello, Natallia Meira Gonsalez, Isabela Pereira Blanco, Marcio Paredes, Leon Alvim, David Machado, Celia Watanabe, Francisco Sergi, Juan Branez, Marcelo Perosa

\section{ABSTRACT}

Introduction: Kidney transplantation (KT) is the therapy of choice for end-stage renal disease. The universal imbalance between demand for KT and available donors requires the development of new strategies to increase the supply of renal grafts. Among these, there is an increasing living-donor KT and the use of expanded criteria donors (ECD). Grafts with acute kidney injury (AKI) are one of the ECD situations, and its use remains controversial due to the uncertainty of kidney recovery after KT. Rhabdomyolysis (RB) is the cause for 7 to $10 \%$ of grafts with AKI, resulting from the release of myoglobin in the circulation and consequent tubular lesion by various mechanisms. Purpose: Motivated by a recent offer of renal graft with RB and subsequent non-authorization for use by the Organ Procurement Organization, the authors aimed to review the literature about the KT feasibility from deceased donor with RB. Methods: Medline database literature review using specific keywords between 2000 and 2018. Results: Seven articles demonstrated series with $51 \mathrm{KT}$ from RB grafts. Of these 29 donors with RB, $20.7 \%$ were on hemodialysis, and the $1.13 \mathrm{mg} / \mathrm{dL}$ mean creatinine. RB grafts presented delayed graft function in $35.3 \%$ of cases, but one month after surgery, kidneys showed complete recovery and normal function, confirmed by biopsy. Discussion: There is great disproportion between the number of patients on the waiting list for kidney and available donors. For this reason, there is need to improve and ease the acceptance criteria of renal grafts either by donors with circulatory death and donors with AKI. RB is one of the causes of AKI among deceased donors. KT from donors with RB showed a higher rate of delayed graft function, but fully recovered around 30 days after transplantation. Conclusion: In Brazil, there are no well-set criteria for using renal grafts with RB, but current studies demonstrate good outcomes and recovery of renal function in the majority of these cases. So far, the use of renal grafts with RB should be at discretion and judgment of each team.

Keywords: Kidney Transplantation; Rhabdomyolysis; Tissue and Organ Procurement; Acute Kidney Injury.

\section{REFERÊNCIAS}

1. Kim WR, Lake JR, Smith JM, Schladt DP, Skeans MA, Harper AM, et al. OPTN / SRTR 2016 Annual Data Report : Kidney. 2016;1-37.

2. Câmara T, Garcia V, Pacheco L. Registro Brasileiro de Transplantes - Veículo Oficial da Associação Brasileira de Transplante de Órgãos. Abto [Internet]. 2017;Ano XXI(No 3). Available from: http://www.abto.org.br/abtov03/Upload/file/RBT/2017/rbttrim3leitura.pdf

3. Klein R, Galante NZ, Franco M, De MCM. Transpondo limites com doadores falecidos : transplantes bem-sucedidos com rins de doador com creatinina sérica igual a $13,1 \mathrm{mg} / \mathrm{dL}$. 2008;133-7.

4. Thomusch O, Gerstenkorn C, Boehm J, Arldt T, Hopt U, Pisarski P. Successful transplantation of kidneys from a donor with myoglobinuric acute renal failure. Am J Transplant. 2006;6(10):2500-1.

5. Boffa C, van de Leemkolk F, Curnow E, Homan van der Heide J, Gilbert J, Sharples E, et al. Transplantation of Kidneys From Donors With Acute Kidney Injury: Friend or Foe? Am J Transplant. 2017;17(2):411-9.

6. Joshi S, Ayyathurai R, Eldefrawy A, Aminsharifi J, Ekwenna O, Sageshima J, et al. Rhabdomyolysis with acute kidney injury in deceased donors is not a contraindication for kidney donation. Int Urol Nephrol. 2012;44(4):1107-11.

7. Leyking S, Poppleton A, Sester U, Ohlmann C-H, Stöckle M, Fliser D, et al. Kidney Transplantation From a Deceased Donor With Anuric Acute Kidney Injury Caused by Rhabdomyolysis. Transplantation [Internet]. 2014;98(10):e87-8. Available from: http://content.wkhealth.com/linkback/openurl?sid=WKPTLP:landi ngpage \&an=00007890-201411270-00019
8. Varrier M, Fisher R, Ostermann M. Acute Kidney Injury -an Update. EMJ Nephrol. 2015;3(1):75-82.

9. Anil Kumar MS, Khan SM, Jaglan S, Heifets M, Moritz MJ, Saeed MI, et al. Successful transplantation of kidneys from deceased donors with acute renal failure: Three-year results. Transplantation. 2006;82(12):1640-5.

10. Molina M, Apaza J, González Monte E, Gutiérrez Martínez E, Sevillano AM, Marín JP, et al. Results of kidney transplantation from deceased donors with acute kidney injury. Transplant Proc [Internet]. 2015;47(1):42-4. Available from: http://dx.doi. org/10.1016/j.transproceed.2014.11.07

11. Guilherme Gomide CABRAL, Leticia Mattar MONICE, Lorena Rocha Dias MACHADO, Luana Modesto Lopes CALDEIRA LR e S\& $\mathrm{H}$ de $\mathrm{AC}$. Insuficiência renal aguda devido à rabdomiólise. Acta Biomed Bras. 2012;3(2):42-7.

12. Phair J, Kayler LK. Transplant of Kidneys from a Small Pediatric Donor with Severe Rhabdomyolysis. Transpl J [Internet]. 2013;96(6):e46-7. Available from: https://fhw1xwvwasp01.fhwa. dot.gov/hfl/innovations/pdfs/uhpc_conn.pdf

13. De Francesco Daher E, Cordeiro NF. Acute renal failure due to rhabdomyolysis. Rev Bras Med. 1998;55(7):18-21.

14. Mekeel KL, Moss AA, Mulligan DC, Chakkera HA, Hamawi K, Mazur MJ, et al. Deceased donor kidney transplantation from donors with acute renal failure due to rhabdomyolysis: Brief communication. Am J Transplant. 2009;9(7):1666-70.

15. Chen C-B, Zheng Y-T, Zhou J, Han M, Wang X-P, Yuan X-P, et al. Kidney transplantation from donors with rhabdomyolysis and acute renal failure. Clin Transplant. 2017;31(8). 